\title{
On a Fundamental Assumption in Linguistics
}

\author{
Mizutani Sizuo
}

The National Language Research Institute, Tokyo

We live in a world of meaning. In most cases, it is as a medium to communicate meaning that we use language. Conversely, the most important role of language is the expression of meaning. When taking linguistic phenomena into consideration, we may not forget the aspect of the linguistic act. "Linguistics" however, has rather been interested in the language as a thing, viz. linguistic signs, specially the vocal signs or their system. In addition, some linguists are inclined to eliminate the viewpoint of meaning from their theories because of its operational difficulty. This tendency naturally accentuates their reluctance toward the language as an act.

They say, of course, that they do not necessarily reject the consideration of meaning in their linguistics. They assume that, among members belonging to the same speech community, linguistic symbols are used in the same, or at least in a similar way. The present writer understands this assumption to imply that the native language is intelligible in the speech community. There is however some doubt whether this assumption can be accepted so easily, though it may seem both preferrable and convenient for the linguists. Once this assumption accepted, linguistic studies can progress smoothly to a considerable extent by considering only formal and meaning-free operations. The present writer as a research worker who takes an interest in machine translation and other information processing by means of electronic computers, accepts the possibility of establishing an algebra of the language. If one pauses however to consider from some distance the beautifully balanced system of linguistic signs, the above assumption remains a problem. Some discussions on this problem will be proposed in this paper.

\section{A Model of the Linguistic Act}

As an introduction, the present writer begs to present his model of the linguistic act.

The language as a thing is a product of linguistic acts. It is through such acts that a speaker or a writer, expecting reception (not always entire understanding) of his expression, shows his hearer or reader some meaning, and that a hearer or a reader, expecting presentation of meaningful contents, tries to surmise them. If one takes away the actors on both sides, there exists no language; there are nothing but physical waves.

We suppose that existence of the following as the necessary condition for the 
act of linguistic expression: the expressing $I^{13}$ namely the transmitter who performs such intended expression, the receiving you namely the receiver ${ }^{2)}$ to whom the transmitter orientates his expression, and the object to be expressed. Similarly for the act of linguistic reception, we suppose the existence of the expressing you, the receiving $I^{3)}$ and the object. Next, a linguistic symbol may be defined as a sort of pattern recognized in the transmitter's or the receiver's linguistic act; it is the name given to the pattern considered as an abstract substance.

We can now proceed in the following way:

i. The language is realized in the act by which an $I$ performs an expression or a reception for a you.

ii. A linguistic field where the $I$ and the you confront each other, is shaped when the $I$ is acting either as a transmitter or as a receiver.

iii. Linguistic expression is an act limited by the transmitter's field, to project the contents of the object to be expressed by means of linguistic symbols. Linguistic reception is an act limited by the receiver's field, to conjecture the original contents by means of the received sequence of symbols.

In other words our hypothesis may be expressed as follows:-

A) We shall distinguish three sorts of sets: (1) a set, $E$, of Event $e$, each of which can be an expressed object either in the real external world or only in the imaginary world; (2) a set, $X$, of Expressed Content $x$ about $e$; (3) a set, $Y$, of Sequence $y$ by linguistic symbols.

B) There is a mapping $\mu$ of $E$ into $X$ and its inverse $\mu^{-1}$, both of which are not always unique.

There is a transformation $\varphi$ of $X$ onto $X$ and its inverse $\varphi^{-1}$. We may assume the existence of the singular $\varphi_{0}$ such that $x \varphi_{0}=x$ holds for any $x$.

There is a mapping $\psi$ of $\{x \psi\}=X$ into $Y$ and its inverse $\psi^{-1}$, both of which are not always unique.

One can foresee that the totality of these correspondences will be a very complicated system. It is not clear at present whether its complete formalization will ever be possible. However, the above scheme opens wide possibilities for linguistic studies.

At first, the linguistic expressing act is an operation defined by

1) The terms "expressing I" and "receiving you" seem somewhat strange. By these terms the present writer aims to emphasize that the level of our discussion is on the viewpoint of the persons concerned, not of an observer in other words a linguist observing the act. (This idea originates from ToKIEDA's theory. Cf. ToKIEDA Motoki, Kokugogaku Genron, Tokyo, 1946). The Japanese equivalents are syutai and aite respectively. It may be unavoidable that in social sciences, theories are influenced by the mother tongue of the scientist. For instance, F. de SAUSSURE contributed to modern linguistics by the establishment of a distinction between langue and parole. He owed this to the fact that the French language happens to possess such words as langue, parole and langage.

2) In talking to oneself, of course, one as a transmitter speaks to himself as a receiver, on the other hand.

3) This may be other than the original receiver, for instance when there is a third party's interpretation, in which case the interpreter is the receiving $I$. 


$$
y=((e \mu) \varphi) \psi=(x \varphi) \psi=x(\varphi \circ \psi) \text { or } e(\mu \circ \varphi \circ \psi),
$$

and it is an expressing $I$ who applies this product of mappings to $e$. The second mapping $\varphi$ may be interpreted as restriction of the field (or bamen no seiyaku as it is called by ToKLEDA). The third mapping $\psi$ is a pattern of linguistic habits by which the transmitter expresses meaning. The order recognized in a set of $\psi$ shall be called a linguistic system. One may notice that $I$ have defined the linguistic system not on the level of signs but on the level of patterns of acts. The linguistic system, of course, can also be defined from the side of the receiving act. Notice by the way, that a linguistic symbol is either $y$ itself or each fragment obtained by an appropriate partition of $y$. Mapping $\mu$ will be mentioned later.

Secondly, the linguistic receiving act for a given $y$ is defined by

or

$$
y(\varphi \circ \psi)^{-1}=y\left(\psi^{-1} \circ \varphi^{-1}\right)=\left(y \psi^{-1}\right) \varphi^{-1}=x^{\prime} \varphi^{-1}=x
$$

$$
y(\mu \circ \varphi \circ \psi)^{-1}=\left(\left(y \psi^{-1}\right) \varphi^{-1}\right) \mu^{-1}=e,
$$

and it is a receiving $t$ who applies this product of mappings to $y$.

Now, which is to be chosen as the terminal point of receiving act, $x$ or $e$ ? To answer this question, one has to consider the problem of the field of linguistics.

\section{Field of Linguistics}

If somebody takes literally a sentence like: "Aren't you smart?", this can be explained by saying that, in spite of his correct choice of $\psi^{-1}$, he has failed in the choice of $\varphi^{-1}$-he has applied a $\varphi_{0}^{-1}$, different from the inverse of the original $\varphi_{1}$ which was used by the transmitter. In the case of this example, we need not return to the level of $e$ for the proof that he misunderstood. Next, supposing the phrase "There's a smart member on the committee": and supposing that a receiver realize it as an ironical expression. Even so the receiver may or may not understand sufficiently, because the above sequence leaves room for various interpretations, under the condition it is understood as irony. For instance, «There is a foolish member on the committee $\gg, \ll A$ certain member on the committee is foolish $\gg, \ll M r$ So-and-so is foolish》 and so forth. There refer to different objects respectively. If not one among them can be specified, the receiver can not be regarded as successful in this case.

This is where the opinions start to diverge. If one considers the above three interpretations between quotation marks as elements of the set $X$, then the linguist is not bound by the mapping $\mu$ and in consequence, neither by the set $E$. It can be affirmed that both $E$ and $\mu$ are outside the language. On the contrary, if one considers the above contents as elements of the set $E$, then $E$, or at least $\mu$, lies within the field of linguistics. ${ }^{4)}$

Thus we may suggest the possibility of various kinds of linguistics as follows: 1. A linguistic which would have for object $Y,\{\psi\},\{\varphi\}, X$ and $\{\mu\}$ for any

4) I do not affirm here that, from this point of view, the study of $E$ itself and of its elements belongs to linguistics. 
given $E$.

2. A linguistic which would have for object $Y,\{\varphi\}$ and $\{\varphi\}$ for any given $X$.

3. A linguistic which would have for object $Y$ and $\{\psi\}$ for any given $\{x \varphi\}$.

4. A linguistic exclusively concerned with the structure of a given $Y$.

Phonetics and phonemics can be studied as part of the above Linguistic 4 . Even grammer and lexicology can be studied as Linguistic 4, in so far as they are regarded as theories of a system of symbols as things. It also will be possible to treat machine translation from this point of view, stipulating appropriate correspondences between two linguistic systems $\mathrm{L}_{1}$ and $\mathrm{L}_{2}$. One goal of this Linguistic 4 is to erect an algebra of linguistic symbols.

On the other hand, Linguistic 4 is not concerned with language or meaning in the aspect of human acts. For this purpose we must adopt at least Linguistic 3. In my opinion, grammar, lexicology and even machine translation is mainly to be studied on this level. Moreover, Linguistic 3 is not sufficient for the study of the so-called keigo (honorific language). The Japanese language has many patterns to express the same content, say, Tarô saw Hanako's garden, such as

Tarô wa Hanako no niwa o mita;

Tarô wa Hanako no niwa o mimasita;

Tarô-sama wa Hanako no niwa o goran ni natta;

Tarô wa Hanako-sama no oniwa o haiken sita;

Tarô-sama wa Hanako-sama no oniwa o haiken nasatta;

Tarô-sama wa Hanako-sama no oniwa o gora ni narimasita; etc.

What differences are recognized among these patterns, and what conditions determine the selection among such-and-such patterns-these problems remain unsolved if one does not refer to the mapping $\varphi$. Thus, it is Linguistic 2 that puts $\{\varphi\}$ within the field of linguistics. Take stylistics for other instance. It is clear that stylistics is concerned with $\varphi$. In addition, it seems to be necessary for stylistics sometimes even to return to the set $E$.

We must now call attention to the fact that Linguistic 2 is not enough for problems as: What kind of linguistic act expresses a lie; How to determine the truth of a received sequence $y$; How to read the transmitter's (the expressing YoU's) mind; How to shape an expression with the certitude of being understood, etc. We have reached Linguistic 1 at last.

These four types of linguistics are equally concerned with the study of language. This is even true of Linguistic 1. Among the four kinds of linguistics enumerated above may be said that it is Set $Y$ that could be said to have a monopoly. However, the linguistic act itself is the process. Either $e \rightarrow y$ or $y \rightarrow e$. Therefore the science of language in the broadest sense is Linguistic 1 , and in the narrowest sense Linguistic 4. I can find no persuasive reason why one of these and one only should be the only proper linguistics. To preserve a theoretical elegance should one keep linguistic so narrow as to limit its scope? The present writer intends to show now in Sections 3 and 4 , that the fundamental assumption to maintain the purity of linguistics is not a safe thing to do. Of course he recognizes also that the lower the number given to the types of lin- 
guistics, the more difficult it becomes for the so-called linguists, to treat the problems.

\section{On the Probability That a Correct Understanding Occurs}

It is the sequence of linguistic signs that is the most important key given directly to the linguist, whichever viewpoint he may choose. He must start form each sequence $y$, and try to puzzle out its meaning. Not any sequence can be used as the material data of his analysis, unless he has made certain that it is indeed a sequence in this linguistic system he is going to analyze and that is done by understanding its meaning. Interpretation precedes in linguistics. The same thing can be said for experimental research. He must interpret the linguistic meaning of the obtained responses. Unluckily, we sometimes make mistakes in interpretation. Therefore the fundamental assumption that in a speech community linguistic symbols are always used in an identical way has to be submitted to critical appraisal. For this purpose we draw the attention to the following simple model of linguistic communication process. ${ }^{\text {s) }}$

We do not always say exactly nor frankly what we think. So we need various techniques of expression and of reception. Let us consider a simple example, in which there are only two expressed contents $x_{1}$ and $x_{2}\left(=\right.$ non- $\left.x_{1}\right)$, and only three sequences $y_{1}, y_{2}$ and $y_{3}$ :

$x_{1}=\mathrm{I}$ know the true state of affairs,

$x_{2}=\mathrm{I}$ do not know the true state of affairs;

$y_{1}=I$ got at the bottom of the affairs,

$y_{2}=I t$ 's very difficult to know the actual state of affairs, $y_{3}=I$ don't know the actual state of affairs.

The transmitter A does not always send $y_{1}$ for $x_{1}$. He may choose $y_{2}$ or $y_{3}$, if he believes $y_{2}$ or $y_{3}$ is more preferable in his situation. But the probability that he chooses a certain $y$ for a certain $x$ may be (almost) stable for any $x$ and any $y$, against a similar receiver in a similar situation. We shall assume this. Then the expressing act, now simply the mapping of $\{x\}$ onto $\{y\}$, can be written as a $(2,3)$ probability matrix

$$
T=\left[\begin{array}{lll}
t_{11} & t_{12} & t_{13} \\
t_{21} & t_{22} & t_{23}
\end{array}\right] \text { or }\left[t_{i j}\right] \text { simply, where } t_{i j}=\operatorname{Pr}\left\{y_{j} \mid x_{i}\right\} \text {. }
$$

Similarly the receiving act that Receiver $B$ surmises Transmitter A's $x$ by the given $y$ under the condition that the medium-spatial process is noiseless, can be written as a $(3,2)$ matrix

$$
R=\left[\begin{array}{ll}
r_{11} & r_{12} \\
r_{21} & r_{22} \\
r_{31} & r_{32}
\end{array}\right] \text { or }\left[r_{j k}\right] \text { simply, where } r_{j k}=\operatorname{Pr}\left\{\hat{x}_{k} \mid y_{j}\right\},
$$

and where $\hat{x}_{k}$ denotes an interpreted content by $\mathrm{B}$ distinguished from the original

5) Details were given in MizUTANI (1962), Outline of a theory of verbal communication process, Keiryô Kokugogalcu (Math. Ling.) No. 21. 
$x_{i}$ by A. Besides we supposed, for the sake of simplicity, that there is no case of impossibility of understanding. This stipulation does not interfere with the point of our discussion here. Then, putting $A=\left[\begin{array}{ll}a_{1} & a_{2}\end{array}\right]$, where $a_{i}$ is a probability $\operatorname{Pr}\left\{x_{i}\right\}$ that $x_{i}$ is adoptted as expressing a content by the transmitter, the expression-interpretation process can be generally written as

$A T R$.

We shall introduce a new matrix $H$ defined as the product of $T R$, which is given by

$$
\begin{aligned}
& H=\left[h_{i k}\right], \quad \text { where } h_{i k}=\operatorname{Pr}\left\{\hat{x}_{k} \mid x_{i}\right\} \text {; } \\
& =\left[\begin{array}{ll}
h_{11} & h_{12} \\
h_{21} & h_{22}
\end{array}\right] \quad \text { in our case. }
\end{aligned}
$$

All elements of the principal diagonal of $H$ take the form $h_{i i}$, where $h_{i i}=\operatorname{Pr}\left\{\hat{x}_{i} \mid x_{i}\right\}$. Therefore it is very natural to say that they correspond to a correct understanding. Thus we shall stipulate:

$1^{\circ}$ The receiver's understanding is said to be correct if and only if $\hat{x}=x$ holds.

$2^{\circ} \quad \hat{x}_{k}=x_{i}$ holds if and only is $k=i$ holds.

Any element $h_{i i}$ gives the rate of correct understanding for the specified content $x_{i}$ (according $\hat{x}_{i}$ ). Then we shall define $G$, which is generally an $(m, 2)$ matrix where $m$ denotes the number of rows of $T$, as

$$
G=\left[\begin{array}{ll}
h_{11} & 1-h_{11} \\
h_{22} & 1-h_{22} \\
\ldots \ldots & \ldots . . \\
h_{m m} & 1-h_{m m}
\end{array}\right], \quad \text { or }\left[\begin{array}{ll}
h_{11} & 1-h_{11} \\
h_{22} & 1-h_{22}
\end{array}\right] \text { in our case. }
$$

Multiplication $A G=F=\left[f_{1} f_{2}\right]$ gives the average rates of correct understanding and misunderstanding: where

$$
\begin{aligned}
& f_{1}=\sum_{i} a_{i} h_{i i}=\sum_{i} \sum_{j} a_{i} t_{i j} r_{j i}, \\
& f_{2}=\sum_{i} a_{i}\left(1-h_{i i}\right)=1-f_{1} .
\end{aligned}
$$

If this $f_{1}$ is (approximately) equal to 1 for any cases, we can adopt the abovementioned fundamental assumption without hesitation. Is it so? Some numerical examples will be shown below.

At first we shall specify matrices $A, T$ and $R$ as follows:

$$
A=\left[\begin{array}{ll}
0.4 & 0.6
\end{array}\right], \quad T=\left[\begin{array}{lll}
0.6 & 0.3 & 0.1 \\
0.1 & 0.4 & 0.5
\end{array}\right] \text { and } R=\left[\begin{array}{ll}
0.8 & 0.2 \\
0.3 & 0.7 \\
0.1 & 0.9
\end{array}\right] \text {. }
$$

The average rate of correct understanding calculated by Eq. (6) for these matrices, is $68.2 \%$. This rate is not very high. The result becomes a cause of concern when the receiver is a linguist and the transmitter is his informant.

Next, fixing $T$ and $R$, we shall let the value of $a_{1}$ run from 0 to 1 , when another element $a_{2}$ of $A$ is $1-a_{1}$. The result in this case is shown in Fig. 1. The highest rate is $75 \%$, which can be obtained when $a_{1}$ is equal to 0 ; the lowest rate is $58 \%$, which can be obtained when $a_{1}$ is equal to 1 . The average rate of correct 


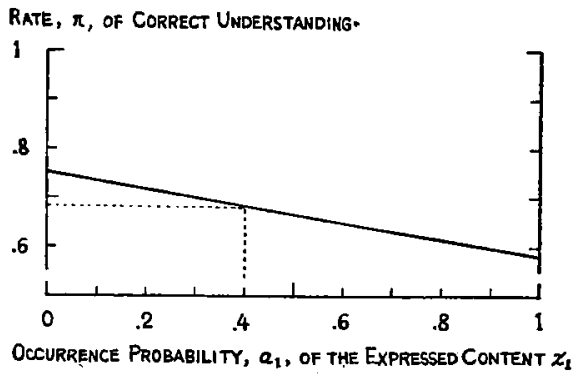

FIG. 1

understanding in repeated observations, is not over $75 \%$.

We have considered the case of a given $R$. Matrix $R$, however, represents the linguist's receiving act. Can he not make the rate higher by an imprevement of his $R$ ? The solution of this problem can be given by BAYES strategy as follows: Make a matrix $U$, each element, $u_{i j}$, of which is $a_{i} t_{i j}$, in other words $\operatorname{Pr}\left\{x_{i} \wedge y_{j}\right\}$; Let the sum of $r_{j i}$ 's corresponding to the maximal $u_{i j}$ 's be 1 , and the others be 0 , for each $j$; Thus $f_{1}$ takes the maximum value, namely the receiver can expect his highest rate of correct understanding. $\bar{R}$ shall denote the matrix $R$ obtained by BAYES strategy. For the practical salution, of course, the receiver must surmise both $A$ and $T$ used by his transmitter. This condition is important for our discussion in Section 4.

Let us return the above-mentioned numerical example. $\bar{R}$ for $A$ and $T$ given in (7) is $\left[\begin{array}{ll}1 & 0 \\ 0 & 1 \\ 0 & 1\end{array}\right]$, and, with $A T \bar{R}$, the improved rate is $78 \%$ (cf. $68.2 \%$ with the original $A T R$ ). In order to know the highest rate by $\bar{R}$, we let the value $a_{1}$ run from 0 to 1 , again. In the case $\vec{R}$ is determined as

$$
\begin{aligned}
& {\left[\begin{array}{ll}
0 & 1 \\
0 & 1 \\
0 & 1
\end{array}\right] \text { if } 0 \leqq a_{1} \leqq \frac{1}{7},} \\
& {\left[\begin{array}{ll}
1 & 0 \\
0 & 1 \\
0 & 1
\end{array}\right] \text { if } \frac{1}{7} \leqq a_{1} \leqq \frac{4}{7},} \\
& {\left[\begin{array}{ll}
1 & 0 \\
1 & 0 \\
0 & 1
\end{array}\right] \text { if } \frac{4}{7} \leqq a_{1} \leqq \frac{5}{6},} \\
& {\left[\begin{array}{ll}
1 & 0 \\
1 & 0 \\
1 & 0
\end{array}\right] \text { if } \frac{5}{6} \leqq a_{1} \leqq 1 .}
\end{aligned}
$$

When $a_{1}$ is exactly equal to $\frac{1}{7}, \frac{4}{7}$ or $\frac{5}{6}$, $\bar{R}$ is not uniquely determined, but both solutions give the equivalent rate in our example. The result is shown in Fig. 2. As seen in the figure, the highest rate is 1 , which is obtained at the point of $a_{1}=0$ or 1 . This means that the receiver using $\bar{R}$ can gain the $100 \%$-rate of correct

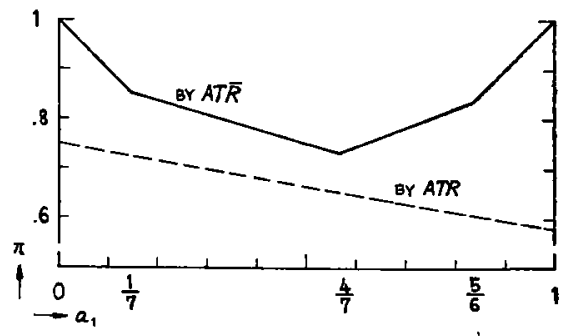

Fis. 2 
understanding only under the condition that the transmitter/always chooses either content $x_{1}$ or $x_{2}$. It may be said to be a trivial case. If $a_{1}$ is equal to $\frac{4}{7}$, the rate even by $\bar{R}$ is no more than $\frac{51}{70} \fallingdotseq 73 \%$.

On our simple example, the probability of correct understanding is not as high that we expect before our analysis.

\section{Criticism of the Fundamental Assumption}

Before proceeding to our critical evalution, we need to examine the validity of the above model. Our premises in Section 3 were as follows:

0) Our principle is based on the model in Section 1 .

1) The number of elements belonging to Set $X$ or $Y$ is very small: $X=\left\{x_{1}, x_{2}\right\}$ and $Y=\left\{y_{1}, y_{2}, y_{3}\right\}$.

2) The spatial process is noiseless, namely any sequence $y$ is not distorted on the way to the receiver.

3) Interpretation is independent from expression expect using a received $y$ as a medium.

4) There is no impossibility of interpretation.

5) Correct understanding is defined by $\hat{x}_{k}=x_{i}$ for an interpreted content $\hat{x}_{k}$ and an expressed content $x_{i}$.

6) $T$ and $R$ are specified by the above (7).

Comparing our model with real linguistic acts, Premise 5) only is too strong, while Premises 1) and 4) are positively advantageous. Generally speaking, our model may be said more advantageous than real linguistic acts.

Nevertheless the rate of correct understanding is mostly rather low as seen in Section 3, and so is the maximum rate by $\bar{R}$. Moreover, in order to obtain such $\bar{R}$, we must add a premise:

7) It is always possible for the receiver to surmise correctly $A$ and $T$ used

by the transmitter,

which is also more advantageous than real acts. Anyhow, the maximizing strategy for the receiver's (in our discussion the linguist's) side, requests to stand at least on the level of $X$. If a linguist wants to stay rigorously within the scope of Linguistic 4 above-mentioned, he must prove that his data are always treated correctly without any refference to meaning. One can see that it is very difficult. That the viewpoint of Linguistic 4 has produced many practical achievements, does not mean anything here, since we are now discussing the principle or recognition in linguistics.

The fundamental assumption then appears on the stage to reply to the above question. However, we have already seen that the possibility of correct understanding is unexpectedly small. Hence, if we wish to keep the assumption, we must stick to the following opinion, namely that interpretation when done literally means also correct understanding, whereas in the case of receiving an unknown language, the understanding is impossible. Some linguists may assert that they 
must not be concerned with more than this. But they argue in circle.

On the fundamental assumption the present writer did not quote any statement directly from existing linguistic literature. He intended to question not such-and-such formula, but the idea behind the formulas. There is no linguist who declears meaning is negligible in any part of linguistics, although one can be found a tendency which practically comes back to such declaration. And this attitude leaves the problem discussed in this paper untouched. Linguists, the present writer included, must grapple with this problem more consciously, whether they give whole-hearted support to the fundamental assumption or not. 